\title{
A Rare Case of Irinotecan-induced Interstitial Pulmonary Disease and Diffuse Alveolar Hemorrhage in a Patient with Pancreatic Cancer
}

\author{
Lingbin Meng ${ }^{1}$, Bo Deng ${ }^{2}$, Baoqiong Liu ${ }^{2}$, Umair Majeed ${ }^{2}$, Wen Wang ${ }^{2}$ \\ 1. Internal Medicine, AdventHealth Winter Park, Winter Park, USA 2. Internal Medicine, AdventHealth \\ Orlando, Orlando, USA
}

$\square$ Corresponding author: Wen Wang,wendysqlwwb@gmail.com

Disclosures can be found in Additional Information at the end of the article

\section{Abstract}

Irinotecan is a cytotoxic medication used to treat various cancers. Diffuse alveolar hemorrhage (DAH) and interstitial pulmonary disease (IPD) are both serious and life-threatening complications, which are rarely reported to be associated with irinotecan. In our case, however, a pancreatic cancer patient developed both DAH and IPD after administration of irinotecan with fluorouracil (5FU).

Categories: Internal Medicine, Oncology

Keywords: irinotecan, chemotherapy, interstitial lung disease, diffuse alveolar hemorrhage

\section{Introduction}

Irinotecan is a cytotoxic medication classified as a plant alkaloid and topoisomerase I inhibitor. It has been approved for treating various cancers including colorectal, gastric, lung, and brain cancers. Diffuse alveolar hemorrhage (DAH) is a serious and life-threatening condition, which can be caused by systemic autoimmune diseases, toxins, transplantation, and medications. Common culprits include antiplatelet and anticoagulant medications. DAH is rarely associated with chemotherapeutic agents. To the best of our knowledge, only one case of irinotecaninduced DAH has been documented in the literature [1]. Although interstitial lung disease is often related to the use of chemotherapeutic medications. IPD is rarely a side effect of Irinotecan [2-4]. We herein report an interesting case of the development of both DAH and IPD in a pancreatic cancer patient after the administration of irinotecan with fluorouracil (5FU).

Received 06/20/2019

Review began 06/23/2019 Review ended 06/24/2019 Published 06/29/2019

(c) Copyright 2019

Meng et al. This is an open access article distributed under the terms of the Creative Commons Attribution License CC-BY 3.0., which permits unrestricted use, distribution, and reproduction in any medium, provided the original author and source are credited.

\section{Case Presentation}

A 60-year-old male with a history of pancreatic cancer presented to our emergency department with a syncopal episode. He was diagnosed with stage IV pancreatic cancer and was started on chemotherapy with a combination of 5-FU and irinotecan (FLOFRI regimen) three months ago. His last chemotherapy was one week prior to admission. On admission, he was noted to have an oxygen saturation of 75\% on 5L oxygen through a nasal cannula. He was then placed on heated high flow oxygen without improvement. The patient was subsequently intubated for worsening acute hypoxic respiratory failure.

The physical examination showed diffused coarse breath sounds in bilateral lung fields. The complete blood count $(\mathrm{CBC})$ revealed that the white blood cell count was 12,000 cells/L, with 


\section{Cureus}

$12 \%$ of band cells, $81 \%$ of neutrophils, $1 \%$ of lymphocytes, $1 \%$ of monocytes, and $1 \%$ of eosinophils. A chest X-ray (CXR) showed diffuse bilateral infiltrates (Figure 1). Computed tomography (CT) of chest revealed subtle ground glass infiltrates and reticular markings suspicious for interstitial lung disease, along with two parenchymal nodules in the left lung (Figure 2). Blood cultures were negative. The urinary antigen of Legionella was negative. Bronchoscopy showed diffuse alveolar hemorrhage. Microscopic examination of the alveolar lavage sample showed large amounts of macrophages and red blood cells (Figure 3), confirming the diagnosis of DAH. Serology was negative for human immunodeficiency virus (HIV), viral hepatitis panel, anti-streptolysin $\mathrm{O}$ antibody (ASO), and C-reactive protein (CRP). Autoimmune panel was negative for antinuclear antibodies (ANA), anti-double-stranded DNA (anti-dsDNA), anti-citrullinated protein antibody (anti-CCP), perinuclear anti-neutrophil cytoplasmic antibodies(P-ANCA), anti-neutrophil cytoplasmic antibody (C-ANCA), anti-cardiolipin, antiglomerular basement membrane (anti-GBM), and anti-topoisomerase antibody (antiSCL70). Direct examination and culture of bronchoalveolar lavage fluid were negative for viruses, bacteria, fungi, mycobacteria, and Pneumocystis jirovecii. Serum polymerase chain reaction (PCR) also was negative for Aspergillus, Cytomegalovirus (CMV), P. jirovecii, and fungi. Cardiac ultrasound showed the ejection fraction of $60 \%$ to $65 \%$ with normal left ventricular diastolic function and mildly elevated right ventricular systolic pressure (RVSP) of $41 \mathrm{mmHg}$.

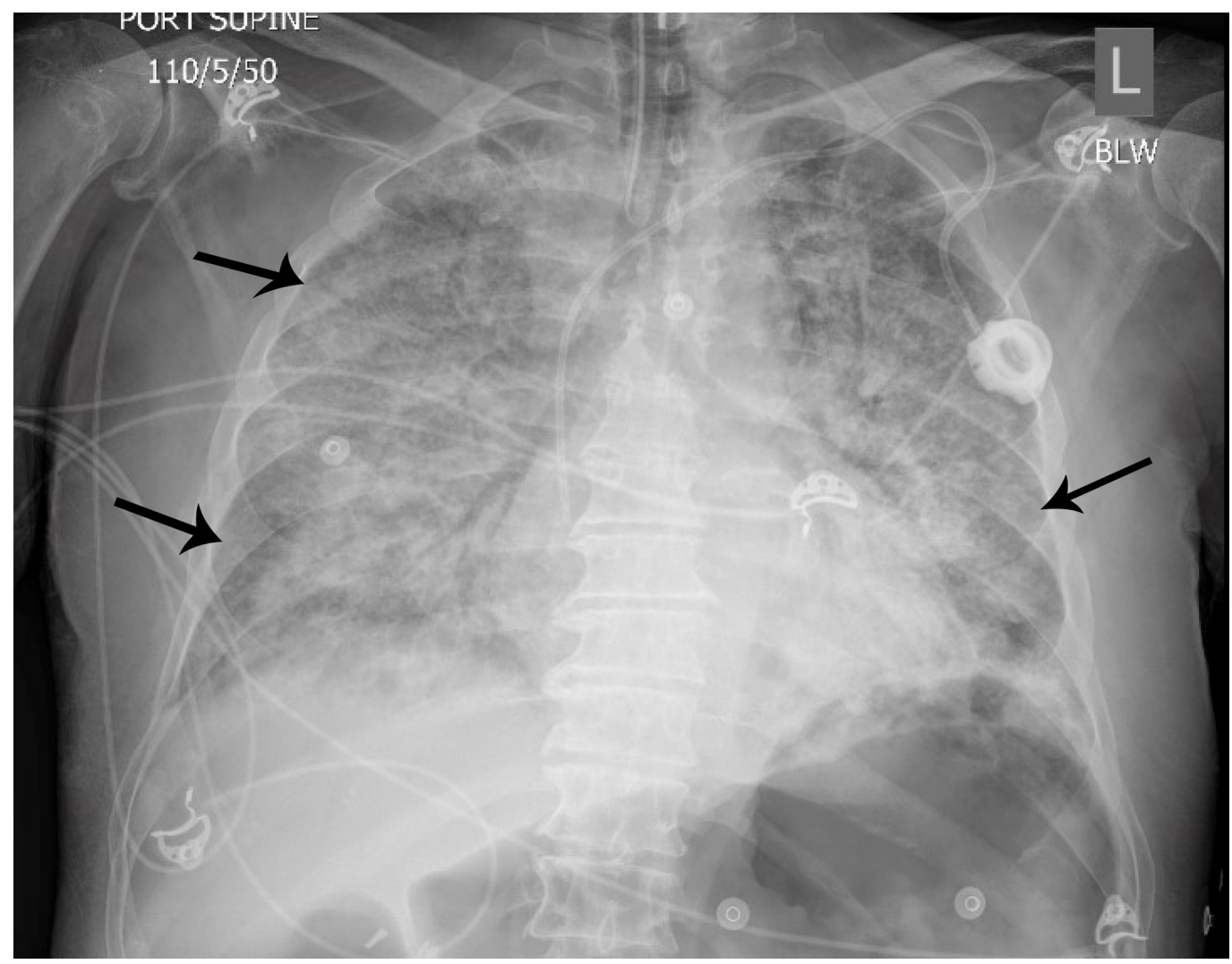

FIGURE 1: CXR shows diffuse bilateral lung infiltrates (black arrows)

CXR, chest X-ray 


\section{Cureus}

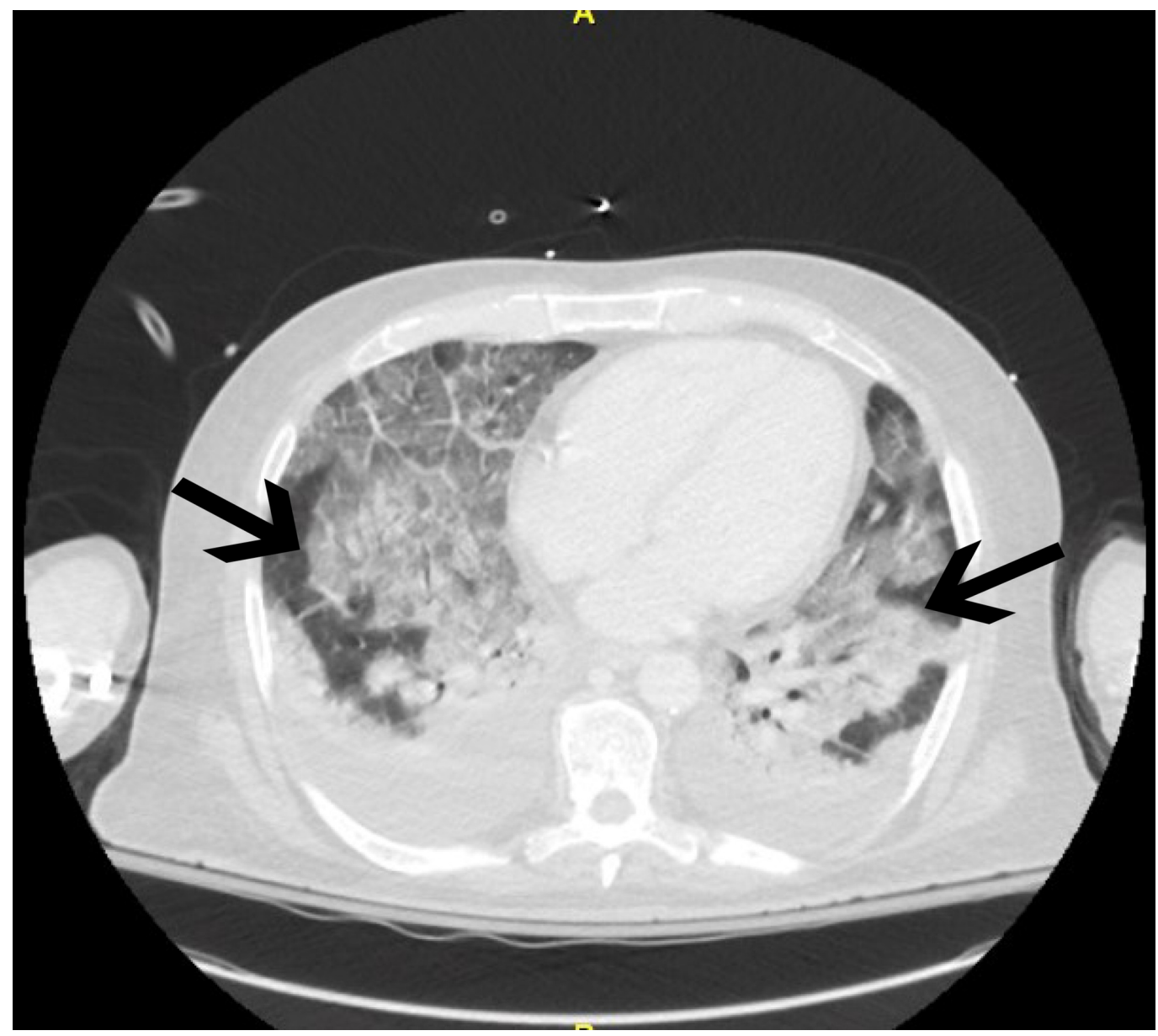

FIGURE 2: CT of chest with contrast demonstrates ground glass infiltrate and increased reticular markings (black solid arrows) in the dependent portion of both lungs suspicious for interstitial lung disease and possible early fibrosis 


\section{Cureus}

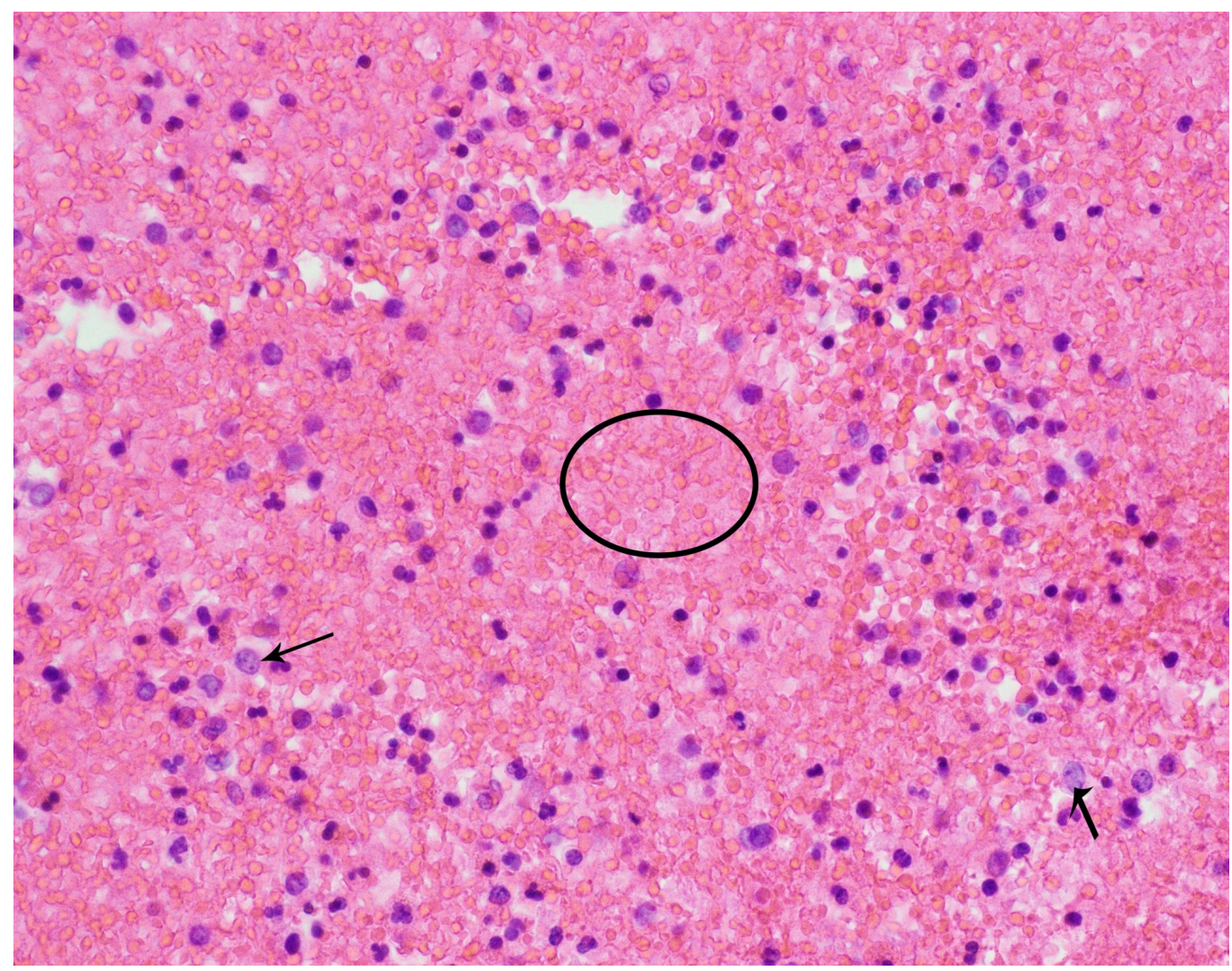

\section{FIGURE 3: Bronchoscopy lavage smear shows scattered macrophages (black arrows) in the background of numerous red blood cells (marked with circle)}

We held chemotherapy in light of the patient's deteriorating condition. We started him on stress dose of steroid and broad-spectrum antibiotics, including cefepime, vancomycin, and azithromycin. The patient required prolonged ventilator support and had to receive tracheostomy as a result. His condition gradually improved after three weeks of supportive treatment. He was eventually extubated successfully. The family decided for hospice care, and the patient was transferred to the inpatient hospice service.

\section{Discussion}

This patient presented with sudden onset of interstitial pneumonia with diffuse alveolar hemorrhage, which was likely caused by his recent administration of the chemotherapeutic agents FLOFRI due to the time coincidence and exclusion of alternative explanations. The patient did not present with any symptoms or signs of connective tissue diseases or systemic autoimmune diseases. Laboratory data was negative for the series of auto-immunological markers. Infection workup was also negative. We did not perform biopsy because the patient was clinically ill and had a significant bleeding risk with a platelet count of 50,000 cells/L. Also, the cytology analysis of bronchial lavage revealed no evidence of cancer metastasis in the lung (Figure 3). Besides chemotherapy with 5FU and Irinotecan, the patient was not on any other medications that may have caused pulmonary toxicities. Overall, the recent chemotherapy with FLOFRI seemed to be directly associated with the development of IPD and DAH in this patient.

Irinotecan was shown to cause non-specific pulmonary toxicities in previous phase II clinical trials [5]. Interstitial lung disease is a well-known side effect of various chemotherapeutic 
medications. But both IDP and DAH are rare complications of Irinotecan administration. Multiple recent cases of irinotecan-induced interstitial pneumonia in treating colorectal cancers were reported in the literature, demonstrating IPD as one of the potential side effects of Irinotecan [2-4]. Only one previous case of irinotecan-induced DAH has been published, suggesting DAH is an extremely rare side effect of Irinotecan [1]. 5FU could also be the potential cause; however, 5FU has almost never been accompanied by pulmonary toxicities. There has been only one report of pulmonary side effects associated with administration of 5FU [6]. Therefore, we consider irinotecan to be the cause of IPD and DAH in this patient, although the contribution of 5FU cannot be completely excluded.

The underlying mechanism of irinotecan-associated IPD and DAH is largely unknown, although direct cytotoxic effect and inflammatory response, such as hypersensitivity-mediated reaction, may play a role. It was postulated that irinotecan, as an isotopomerase inhibitor, can directly inhibit DNA synthesis or repair, inducing cytotoxic effects in pneumocytes and/or the interstitial cells. The cytology of bronchial lavage in this patient revealed the infiltration of large amounts of macrophages, suggesting the involvement of an inflammatory response. Our patient and previously published irinotecan-induced IPD or DAH patients all required systemic steroid therapy, indicating that longer duration of systemic steroids as an adjuvant therapy with Irinotecan or slower tapering of systemic steroids may reduce the incidence of irinotecaninduced IPD or DAH. Further studies are required to test this possibility and identify the exact underlying mechanisms of irinotecan-induced IPD or DAH.

\section{Conclusions}

To the best of our knowledge, our study is the first case report showing irinotecan could possibly induce both IPD and DAH in a pancreatic cancer patient. DAH is a life-threatening situation that requires immediate discontinuation of Irinotecan therapy and administration of systemic steroids. Longer duration of systemic steroids or slower tapering of systemic steroids may reduce the incidence of serious pulmonary adverse events of irinotecan. It is effective for both IPD and DAH. Further studies are required to clarify whether it is a direct toxic effect or a hypersensitivity-mediated reaction.

\section{Additional Information \\ Disclosures}

Human subjects: Consent was obtained by all participants in this study. Conflicts of interest: In compliance with the ICMJE uniform disclosure form, all authors declare the following: Payment/services info: All authors have declared that no financial support was received from any organization for the submitted work. Financial relationships: All authors have declared that they have no financial relationships at present or within the previous three years with any organizations that might have an interest in the submitted work. Other relationships: All authors have declared that there are no other relationships or activities that could appear to have influenced the submitted work.

\section{References}

1. Kim S-H, Minami S, Ogata Y, Yamamoto S, Komuta K: Diffuse alveolar hemorrhage induced by Irinotecan for a patient with metastatic thymic carcinoma: a case report and literature review. Intern Med. 2017, 56:1697-1700. 10.2169/internalmedicine.56.8349

2. Michielin O, Udry E, Periard D, Matzinger O, Lobrinus J, Stupp R: Irinotecan-induced interstitial pneumonia. Lancet Oncol. 2004, 5:322-324. 10.1016/S1470-2045(04)01471-8

3. Miyake M, Takeda T, Haraguchi N, et al.: A case of rectal cancer completely resected after successful treatment with irinotecan cetuximab-induced interstitial pneumonia with steroid pulse therapy. Jpn J Cancer Chemother. 2015, 42:2334-2336. 


\section{Cureus}

4. Aoyagi $\mathrm{H}$, Ito $\mathrm{H}$, Higuchi $\mathrm{K}$, et al.: A case of fatal interstitial pneumonia during FOLFIRI plus Cetuximab therapy for liver metastasis of colon cancer. Jpn J Cancer Chemother. 2018, 45:5153.

5. Pitot HC, Wender DB, O'Connell MJ, et al.: Phase II trial of Irinotecan in patients with metastatic colorectal carcinoma. J Clin Oncol. 1997, 15:2910-2919. 10.1200/JCO.1997.15.8.2910

6. Andou H, Itoh K, Tsuda T: A case of fluorouracil-induced pneumonitis . Jpn J Thora Dis. 1997, 35:1080-1083. 10.11389/jjrs1963.35.1080 